\title{
The effect of using inconsistent ocean tidal loading models on GPS coordinate solutions
}

\section{Yuning Fu, Jeffrey T. Freymueller \& Tonie van Dam}

\section{Journal of Geodesy}

Continuation of Bulletin Géodésique and manuscripta geodaetica

ISSN 0949-7714

J Geod

DOI 10.1007/s00190-011-0528-1

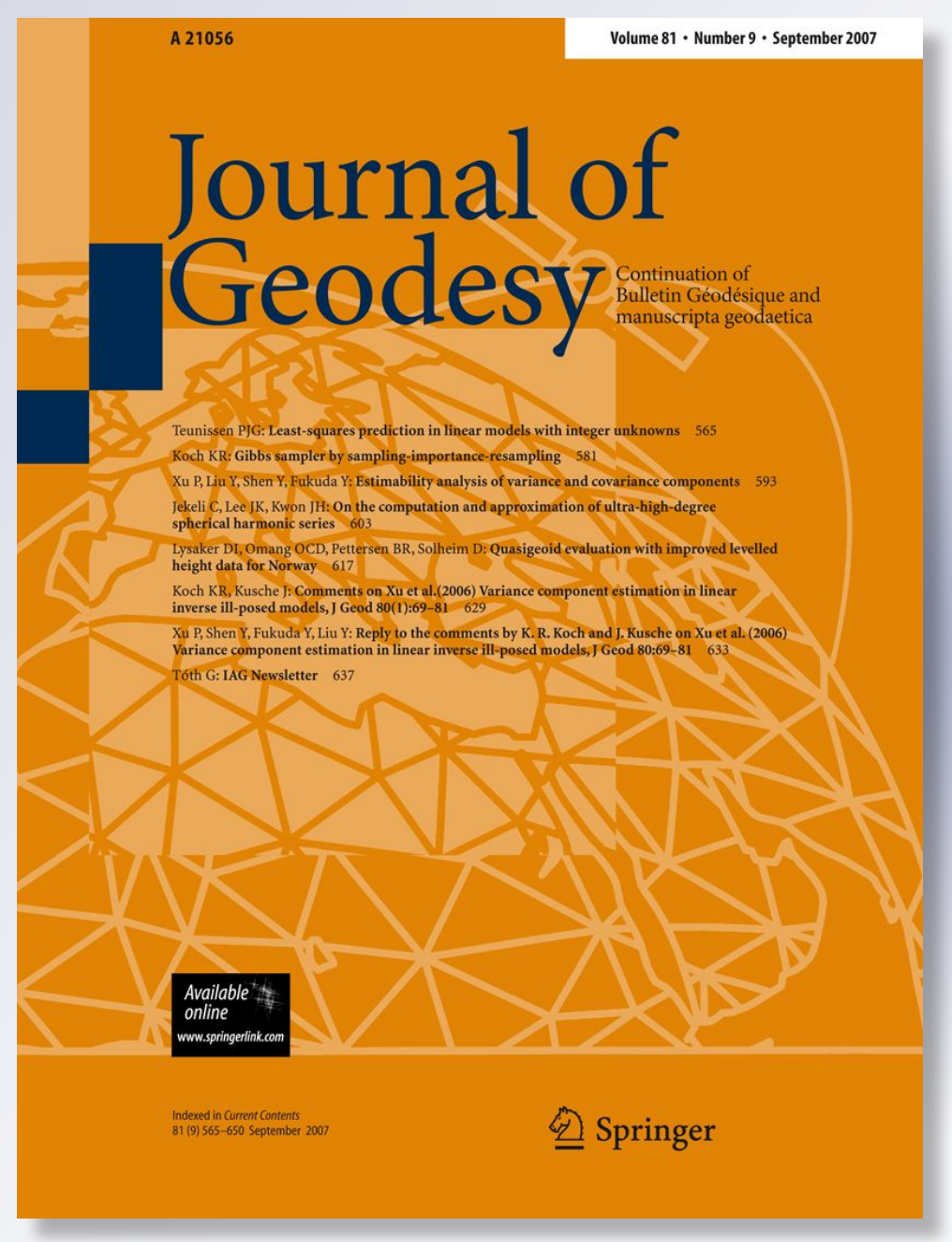

望 Springer 
Your article is protected by copyright and all rights are held exclusively by SpringerVerlag. This e-offprint is for personal use only and shall not be self-archived in electronic repositories. If you wish to self-archive your work, please use the accepted author's version for posting to your own website or your institution's repository. You may further deposit the accepted author's version on a funder's repository at a funder's request, provided it is not made publicly available until 12 months after publication. 


\title{
The effect of using inconsistent ocean tidal loading models on GPS coordinate solutions
}

\author{
Yuning Fu · Jeffrey T. Freymueller · Tonie van Dam
}

Received: 8 February 2011 / Accepted: 3 November 2011

(C) Springer-Verlag 2011

\begin{abstract}
We use up to a 6-year span of GPS data from 85 globally distributed stations to compare solutions using ocean tidal loading (OTL) corrections computed in different reference frames: center of mass of the solid Earth (CE), and center of mass of the Earth system (CM). We compare solution sets that differ only in the frame used for the OTL model computations, for three types of GPS solutions. In global solutions with all parameters including orbits estimated simultaneously, we find coordinate differences of $\sim 0.3 \mathrm{~mm}$ between solutions using OTL computed in CM and OTL computed in CE. When orbits or orbits and clocks are fixed, larger biases appear if the user applies an OTL model inconsistent with that used to derive the orbit and clock products. Network solutions (orbits fixed, satellite clocks estimated) show differences smaller than $0.5 \mathrm{~mm}$ due to model inconsistency, but PPP solutions show distortions at the $\sim 1.3 \mathrm{~mm}$ level. The much larger effect on PPP solutions indicates that satellite clock estimates are sensitive to the OTL model applied. The time series of coordinate differences shows a strong spectral peak at a period of $\sim 14$ days when inconsistent OTL models are applied and smaller peaks at $\sim$ annual and $\sim$ semi-annual periods, for both ambiguity-free and ambiguity-fixed solutions. These spurious coordinate variations disappear in solutions using consistent OTL models. Users of orbit and clock products must ensure that they
\end{abstract}

Y. Fu (凶) · J. T. Freymueller

Geophysical Institute, University of Alaska Fairbanks, Fairbanks, AK 99775-7320, USA

e-mail: yuning@gi.alaska.edu

J. T. Freymueller

e-mail: jeff.freymueller@gi.alaska.edu

T. van Dam

Faculté des Sciences, de la Technologie et de la Communication, University of Luxembourg, Luxembourg, Luxembourg

e-mail: tonie.vandam@uni.lu use OTL coefficients computed in the same reference frame as the OTL coefficients used by the analysis centers that produced the products they use; otherwise, systematic errors will be introduced into position solutions. All modern products should use loading models computed in the CM frame, but legacy products may require loading models computed in the $\mathrm{CE}$ frame. Analysts and authors need to document the frame used for all loading computations in product descriptions and papers.

Keywords Ocean tide loading (OTL) - Reference frame . Geocenter - Global Positioning System (GPS) - Center of mass correction $(\mathrm{CMC}) \cdot$ Power spectrum

\section{Introduction}

Ocean tidal loading (OTL) is the periodic crustal displacement caused by the load of the ocean tides. OTL surface displacements can reach several $\mathrm{cm}$ in magnitude in the vertical component for coastal areas, and are smaller but still detectable in the horizontal components (Vey et al. 2002; Urschl et al. 2005). Additionally, any mismodeling of diurnal and semidiurnal tidal constituents can be propagated to longerperiod signals in the GPS coordinate time series (Penna and Stewart 2003; Penna et al. 2007; Yuan et al. 2009), which results in spurious periodic variations. The magnitude of the OTL deformations makes correcting for them important during precise geodetic data analysis (e.g., van Dam et al. 1997; Dragert et al. 2000; King et al. 2008), especially because the measurement and modeling of variations in time series is becoming more important. Corrections for OTL deformation are usually made by computing a set of coefficients (amplitudes and phases of the loading deformation for each 
tidal component), and subsequently removing this model from the GPS observations in the solution for coordinates and other parameters.

Isomorphic terrestrial reference frames can be defined with differing origins, including the center of mass of the solid Earth (CE), or the center of mass of the whole Earth system (CM), which includes the ocean and other surface loads, such as atmosphere and continental water storage (Blewitt 2003). The International Terrestrial Reference Frame (ITRF) is a CM frame in the sense of long-term secular motions, but does not account for short-term variations, such as seasonal variations or sub-daily variations (Dong et al. 2003). The Center of Figure $(\mathrm{CF})$ frame, defined based on the center of figure of the solid Earth surface, can be well approximated by a no-net-translation condition on a global geodetic network. The $\mathrm{CF}$ frame is very nearly equivalent to $\mathrm{CE}$, with the difference between CEand CF being only about $2 \%$ of the difference between CE and CM (Dong et al. 2003; Blewitt 2003).

Ocean loading corrections can be computed by convolving Green's Functions with tidal variations over the global ocean domain (Goad 1980). Green's Functions are derived from load Love numbers in a specific reference frame, and describe the deformation of the Earth due to a point surface load. The most widely used Green's Functions were obtained by Farrell (1972) in the CE frame. Load Love numbers and the resulting Green's Functions can be transformed between the $\mathrm{CE}$ and $\mathrm{CM}$ frames based on the individual physical definition of geocenter (Dong et al. 1997; Blewitt 2003). The Green's functions differ in that the computed loading differs for the degree 1 deformation. In the CM frame, movement of fluid mass is accompanied by an opposite motion of the center of mass of the solid Earth, while in the CE frame the center of mass of the solid Earth is fixed (by definition). The geocentric component of the frame difference can be on the order of several millimeters in size, depending on how the tidal components add up (Scherneck et al. 2000). (H.-G. Scherneck provides tabulated coefficients for this "center of mass correction" for various tidal models and tidal components at http://froste.oso.chalmers.se/loading/ cmc.html). In this paper, we will use OTL-CM to refer to an OTL model computed in the CM frame, and OTL-CE for a model computed in the CE frame.

The current IERS Conventions and the International GNSS Service (IGS) recommendations both suggest using OTL corrections computed in the CM frame (IERS Conventions 2010; Kouba 2009). However, older conventions were different. Many if not most papers do not specify in which frame their loading computations were calculated, even when OTL and other sub-daily variations are the focus of the paper. For a typical example, King et al. (2008) did not report which frame was used for their OTL computations. But we know they must have used the CE frame Greens functions. This conclusion can only be deduced by a reader who knows that only the CE frame Greens functions were available for the SPOTL code (Agnew 1997) at that time. Few studies have examined the difference between loading computations in the CM vs. CE frame. Scherneck et al. (2000) compared sets of PPP solutions using both OTL-CE and OTL-CM models, and found that the solutions using OTL-CE provided a better match for the tidal variations observed in the solutions. They suggested that this resulted from the fact that the orbit and clock products held fixed in their PPP solutions were generated using OTL-CE models, and that consistency between the solutions that generated the products and the user's solution is important. In this paper, we use PPP and other solutions to evaluate the effects of the frame used for OTL model computations, using an extensive global data set.

The frame used for OTL model computations needs to be kept distinct in the mind from the frame used for orbit integration, or any corrections applied to the orbits in preparing specific file formats. GPS satellites are gravitationally attracted by the mass of the whole Earth system, so their trajectories are physically relative to CM. GPS orbit files in the SP3 format are supposed to be in a crust-fixed reference frame, so the center of mass correction (difference between $\mathrm{CM}$ and $\mathrm{CE}$ ) is removed in preparing these files, at least in the IGS reprocessed products (IERS Conventions 2010). In this paper, we do not discuss frame issues of the orbits themselves, but only the frame used to compute the OTL models. For consistency, all loading computations should be done in a CM frame, but when the frame for the loading computations is commonly not specified, it may be easy for inconsistency to arise, or for an incorrect theory to be applied.

In this study, we reprocessed a multi-year set of GPS data that included 85 globally distributed continuous stations with OTL modeled in both CE and CM, in order to examine the differences when using precise point positioning (Zumberge et al. 1997). We used shorter subsets of this data set for additional, more in-depth comparisons of different types of GPS solutions. In particular, we investigated solution strategies in which (1) the user computes a regional solution using fixed orbit products but estimates satellite clocks; and (2) the user estimates coordinates, orbits and all other parameters in a single solution, rather than using products derived by an external analysis center. We repeated our analysis for two different ocean tidal models and two different OTL computation programs to eliminate the possibility that the observed differences are due to the ocean tide models or to the software used to estimate the corrections.

\section{GPS data and processing strategy}

We analyzed a set of 85 globally distributed continuous GPS stations in this study (Fig. 1). We use a global distribution of 
Fig. 1 Globally distributed continuous GPS stations adopted in this study. We processed 6 years of GPS data using OTL coefficients obtained in different frames. Solid diamonds denote sites used in the stacked power spectral analysis ( $\sim 48$ sites). White diamonds depict stations with nonnegligible temporal gaps or sudden coordinate offsets in the timeseries that were not used in the stack

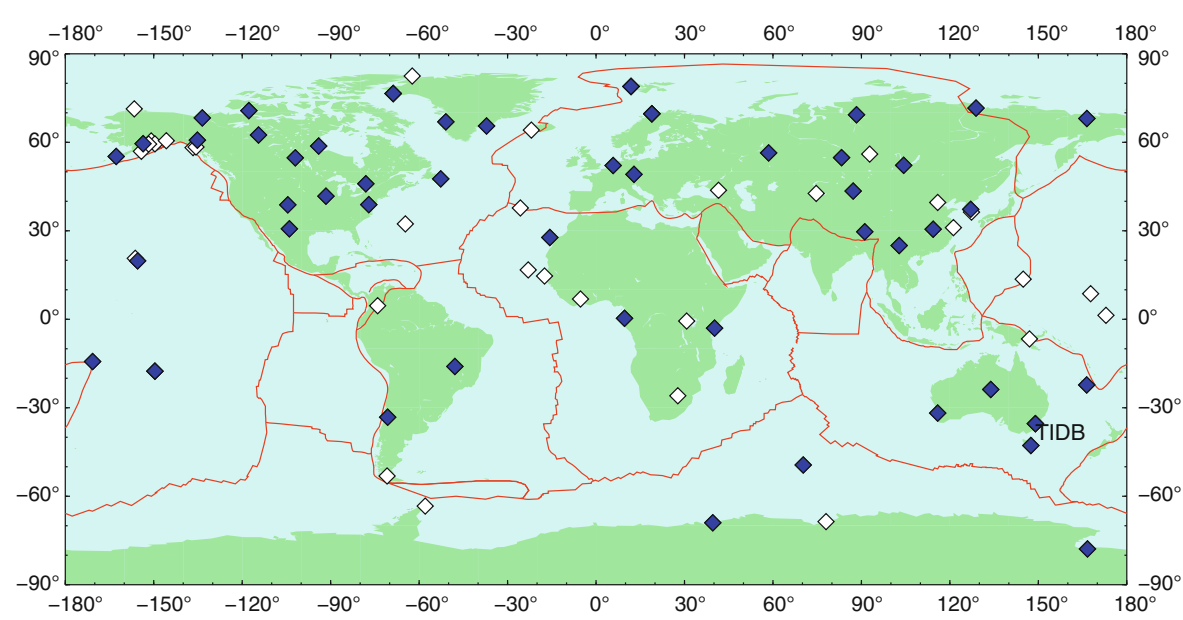

sites because using a regional network may introduce considerable errors during frame alignment (Tregoning and van Dam 2005). We reprocessed all data for these sites from the beginning of 2002 through the end of 2007, 6 years in total, using the GIPSY/OASIS-II (Version 5.0) software in point positioning mode (satellite clocks and orbits fixed) to obtain daily coordinates and covariances. We then analyzed subsets of this data in network positioning mode (satellite clocks estimated, but orbits fixed) and using solutions in which we estimated all parameters simultaneously, including orbits. In point positioning, the user estimates site coordinates using fixed orbit and clock products that were derived by a separate global solution and that are usually provided by an external analysis center. Multiple sites can be analyzed together or one by one, giving the same result either way (Zumberge et al. 1997). We used JPL's reanalysis set of orbit and clock products, which were determined using a consistent set of models over the entire time span, including absolute antenna phase center models for both GPS receiver and satellite antennas (Schmid et al. 2007). We included antenna plus radome specific phase center models where they were available. We used the GMF tropospheric mapping function (Boehm et al. 2006), and adopted a priori dry tropospheric delay estimates from the Global Pressure and Temperature (GPT) model (Boehm et al. 2007). The solutions shown here do not include ambiguity resolution, although we test the impact of ambiguity resolution later in the paper. All solution series used exactly the same data, and in a variety of tests we vary the orbits, clocks, and OTL model used.

For our base set of solutions, we used JPL's fiducial free orbit and clock products (in the native GIPSY format). We transformed each daily solution into the ITRF2005 reference frame, estimating our own frame alignment transformation. Because we transform each solution into ITRF using a global set of reference sites, these comparisons highlight distortions of the network rather than differences in the solutions that can be removed by a 7-parameter transformation. A com- parison of solutions that used the fiducial products that are already in ITRF, would be very similar except that any framelike bias between the solutions would remain as normally no frame alignment transformation is applied to those solutions. In other comparisons, we aligned the OTL-CE solution to the OTL-CM solution using a standard 7-parameter transformation so that we could directly evaluate both distortions and frame-like biases between the solutions.

We used the ocean tide models FES2004 and TPXO7.0 to calculate OTL corrections for all GPS stations, in both the $\mathrm{CE}$ and the $\mathrm{CM}$ frames, using two different software packages. The OTL model amplitudes and phases from the FES2004 tide model (including the tidal components M2, S2, N2, K2, K1, O1, P1, Q1, MF, MM and SSA) were computed using Hans-Georg Scherneck's web tool (http://www. oso.chalmers.se/ loading/). The OTL model amplitudes and phases using TPXO7.0 (Egbert and Erofeeva 2002) (components M2, S2, N2, K2, K1, O1, P1, Q1, MF, MM) were computed using the SPOTL software (Agnew 1997). The Green's functions provided with SPOTL are in the CE frame. We developed our own set of CM Green's functions to use with this program. We compared the SPOTL calculations for TPXO7.0 to those using Scherneck's online tool. The results agreed very closely for both the CE and CM frames (e.g. the difference in amplitude for the M2 component between SPOTL and Scherneck's online tool is less than $1.5 \%$ of its amplitude). This result is consistent with the work of Penna et al. (2008) that also assessed different algorithms for computing OTL.

\section{Comparison results}

3.1 Comparison between solutions using different frames during point positioning

Using JPL's reanalysis orbit/clock products, Fig. 2 depicts the differences between solutions for station TIDB (Canberra, 
Fig. 2 Differences between GPS solutions determined using OTL-CM and OTL-CE coefficients, for the station TIDB. Top 6-year time series of differences using the ocean tide model FES2004. Bottom A 2-year time series comparing the FES2004 (blue) and TPXO7.0 (red) models; at this scale, the two models give nearly identical results
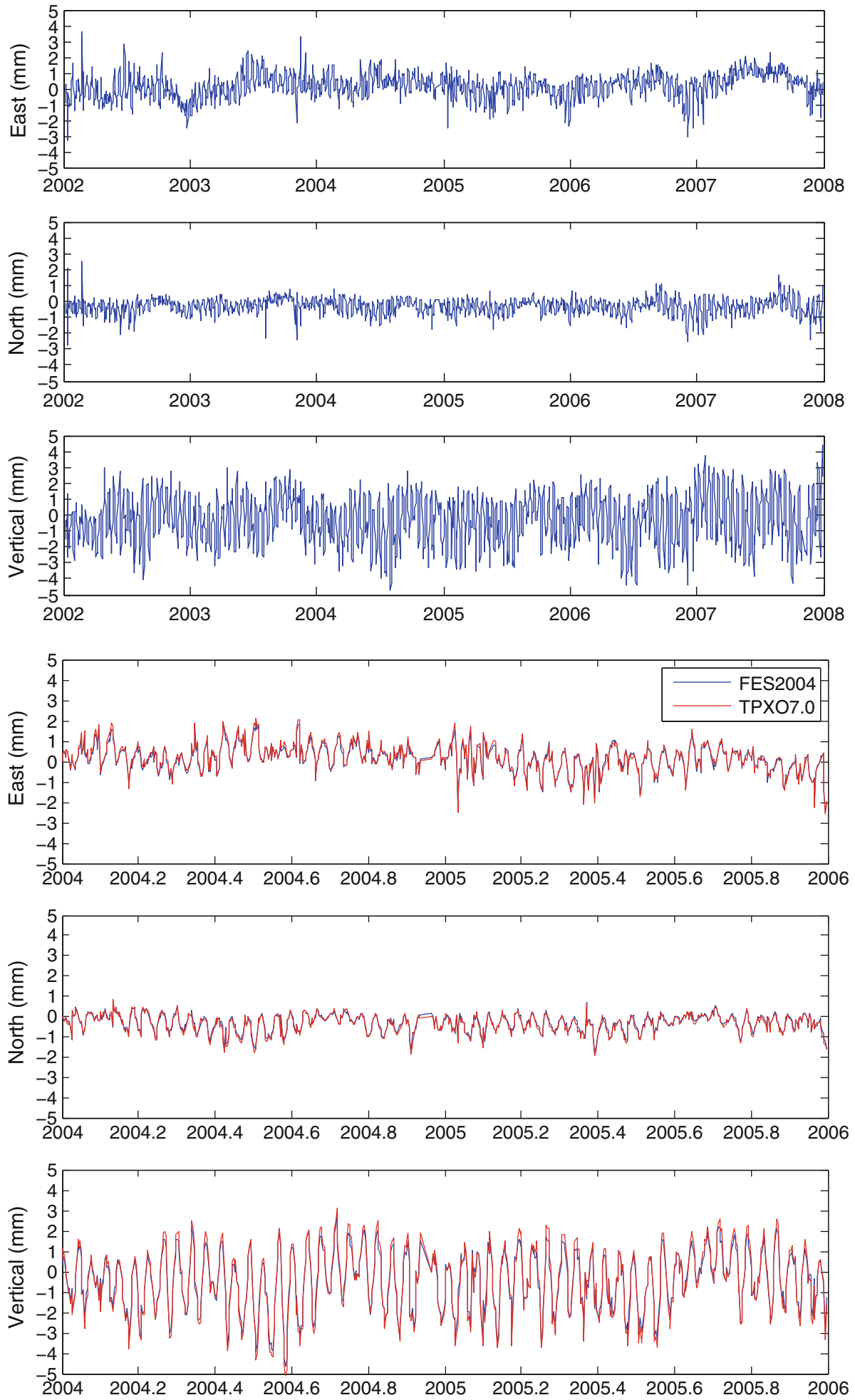

Australia) using OTL derived in the CE frame and the CM frame. The only difference between these solutions is the OTL coefficients used. All the GPS stations investigated in this study, independent of their proximity to the ocean, show very similar patterns. The patterns are similar because the OTL displacements computed in CM and CE differ only by a degree 1 deformation. While all the stations show similar patterns, the phase of peaks of the difference varies over the globe. This result holds for solutions derived using either the FES2004 or TPXO7.0 models, consistent with the conclusion of Thomas et al. (2007) that the present ocean tide models give very similar results at the global scale.

The most obvious periodic component in Fig. 2 has a period of about 14 days, appearing consistently in East, North 


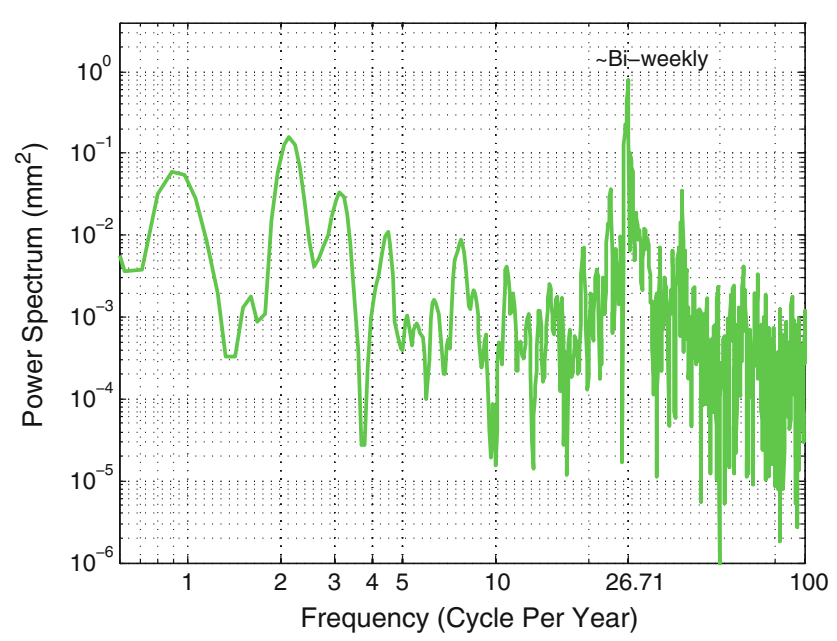

Fig. 3 Power spectrum of vertical coordinate differences from the 6-year timeseries computed using OTL-CM and OTL-CE corrections, for the station TIDB. A sharp peak appears at a frequency of about 26.71 cycles per year. This represents a period of about 13.67 days

and Height. The vertical component has larger peak-to-peak amplitudes, but differences in the horizontal components can reach about one third of that observed in the height. The power spectrum (Fig. 3) for the difference (Fig. 2) shows a spectral peak at 26.71 cycles per year (period of 13.67 days), and also reveals $\sim$ annual and $\sim$ semiannual components. Penna and Stewart (2003); Stewart et al. (2005) and Penna et al. (2007) demonstrated that, with a discrete 24-h GPS data processing strategy, mismodeled diurnal and semidiurnal tidal constituents could be aliased into errors in longer periods in the timeseries. They predicted that propagated tidal deformation would appear at about 14 days (due to the aliasing of M2 and O1), semiannual (due to the aliasing of S2, $\mathrm{K} 2$ and $\mathrm{P} 1$ ) and annual periods (due to the aliasing of S2 and K1). These are the peaks that we observe in the vertical coordinate differences between the two solution sets (Fig. 3). Other studies (Amiri-Simkooei et al. 2007; Ray et al. 2008; Tregoning and Watson 2009) have shown that the observed $\sim$ annual and $\sim$ semiannual peaks in power spectra of GPS timeseries are really GPS draconitic annual ( $\sim 351.4$ days) and semiannual ( $\sim 175.7$ days $)$ periods. A GPS draconitic year is the period for the GPS constellation to repeat its orientation relative to the Sun (Ray et al. 2008). For simplicity, we will refer to these peaks as $\sim$ annual, $\sim$ semi-annual, and $\sim 14$ days or $\sim$ bi-weekly.

Figure 4 depicts the amplitudes of the $\sim 14$-day periodic variation in differences between solutions using OTL-CE and OTL-CM, as a function of latitude. GPS stations located at lower latitudes show larger amplitudes. Similar latitudedependence of the effects of mismodeling signals at tidal frequencies was found by Penna et al. (2007) and Tregoning and Watson (2011).

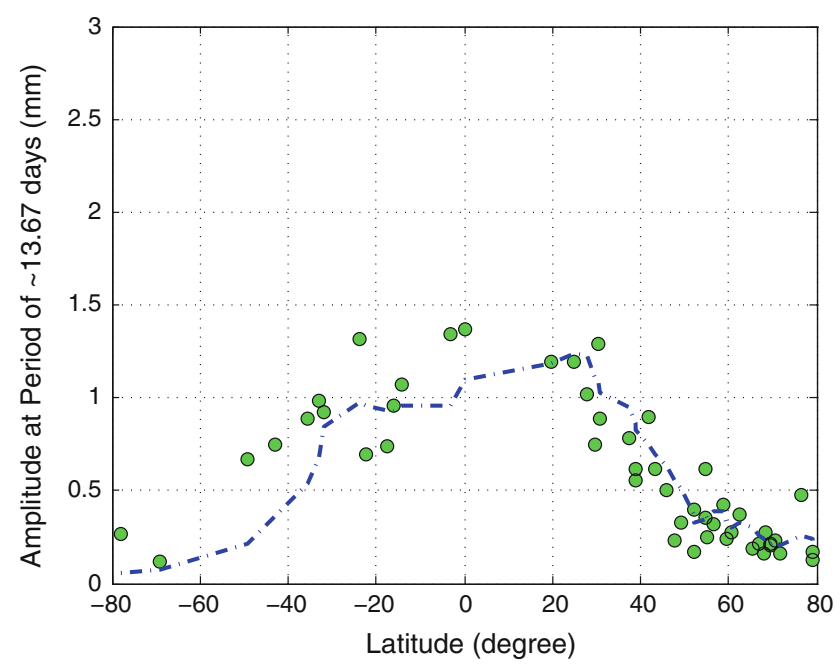

Fig. 4 Amplitude of the $\sim 14$-day periodic variation in the difference between solutions using OTL-CM and OTL-CE coefficients, as a function of latitude. Blue dash-dot line is a five-point moving average

Solutions using OTL-CE and OTL-CM models differ by periodic variations, but which solution contains this spurious periodic variation? The stacked power spectrum for the detrended station timeseries is shown in Fig. 5. The 48 GPS stations used for the stack (solid diamonds in Fig. 1) were chosen based on the criteria that their timeseries should be continuous without obvious gaps or sudden offsets of coordinates due to strong earthquakes or receiver antenna changes. The stacked power spectrum (Fig. 5) clearly illustrates that the $\sim 14$-day periodic variation is present only in solutions using OTL-CE frame. There is no spectral peak around this period in the solutions from the solutions using OTL-CM. This obvious difference between solutions using OTL-CE and OTL-CM does not depend on the tidal model, with similar results using both FES2004 and TPXO7.0 (Fig. 5). The amplitude of the $\sim 14$-day peak in the power spectrum is reduced by $41 \%$ from OTL-CE solutions to OTL-CM solutions for FES2004, and by 40\% from for TPXO7.0. Therefore, the difference between frames is much larger than the difference between ocean load models in the same frame. This result means that the choice of frame for OTL computations is more significant than the choice of ocean tide model. JPL uses the FES2004 ocean tide model and OTL$\mathrm{CM}$ coefficients in the solutions it uses to generate its current and reanalysis orbit and clock products (S. Desai, personal communication 2010). JPL's use of OTL-CM coefficients is consistent with current conventions. Our results show that point positioning users introduce systematic errors into their solutions if they do not use the same OTL coefficients used in the solution that generated the orbit and clock products.

For other periodic components, the amplitudes of the stacked power spectrum for the detrended timeseries derived using OTL-CM are also smaller than those derived using 

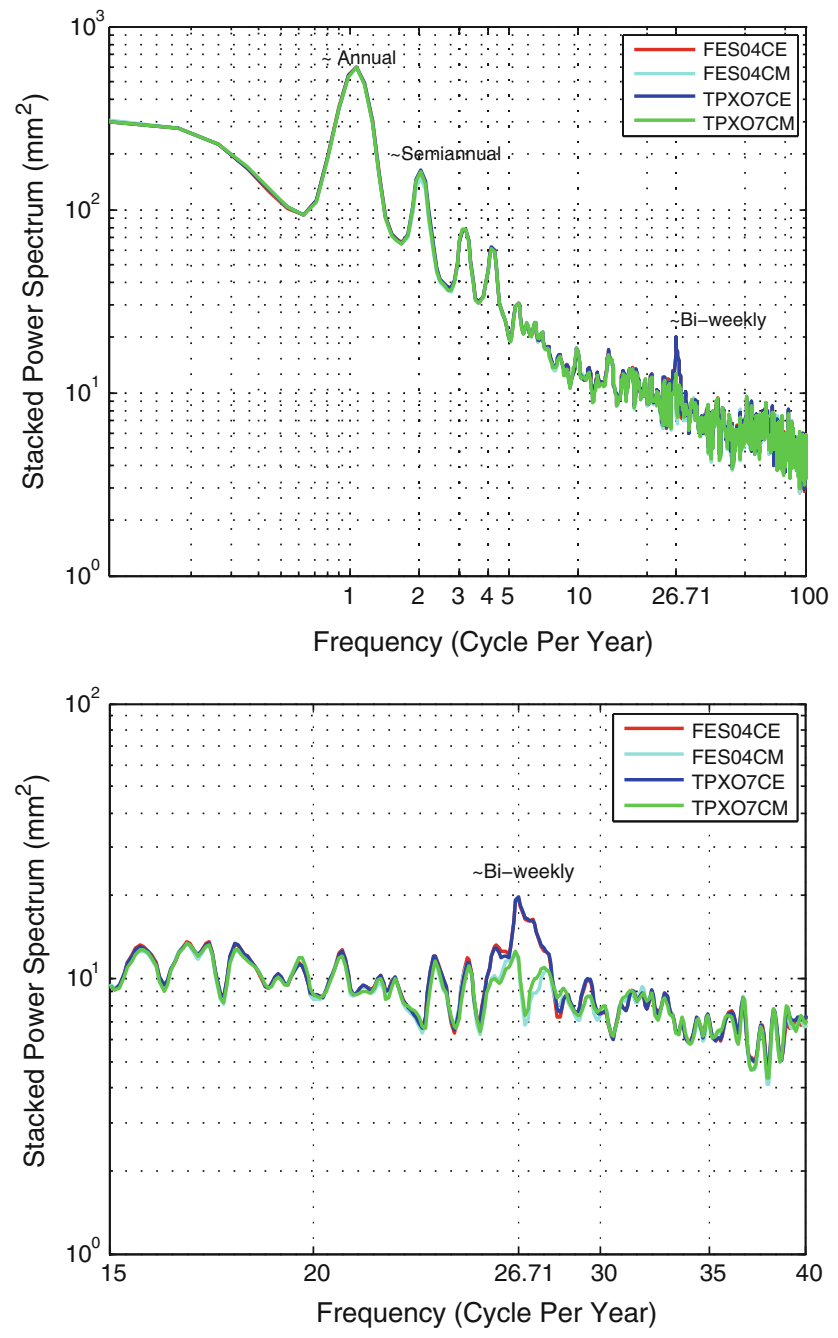

Fig. 5 Stacked power spectrum for the vertical coordinate timeseries, using all stations in the stack (Top). A detailed of the top panel highlighting the $\sim 14$-day period (Bottom). GPS stations used for stack are marked with solid diamonds in Fig. 1

OTL-CE. For the annual component, the amplitude is decreased by $1.13 \%$ by changing from OTL-CE to OTL-CM for FES2004, and by $1.06 \%$ for TPXO7.0. The semiannual is reduced by $3.25 \%$ by changing from OTL-CE to OTL-CM for FES2004, and by 3.44\% for TPXO7.0.

\subsection{Testing the importance of consistency in OTL coefficients}

JPL currently uses OTL-CM coefficients in the solutions that generate its products, but the older JPL legacy orbit products employed OTL-CE coefficients (S. Desai, personal communication 2010). We use this difference to test the hypothesis that the differences we observe result from inconsistencies between the user solution and the solution that generated the orbit and clock products. We reprocessed data for all of

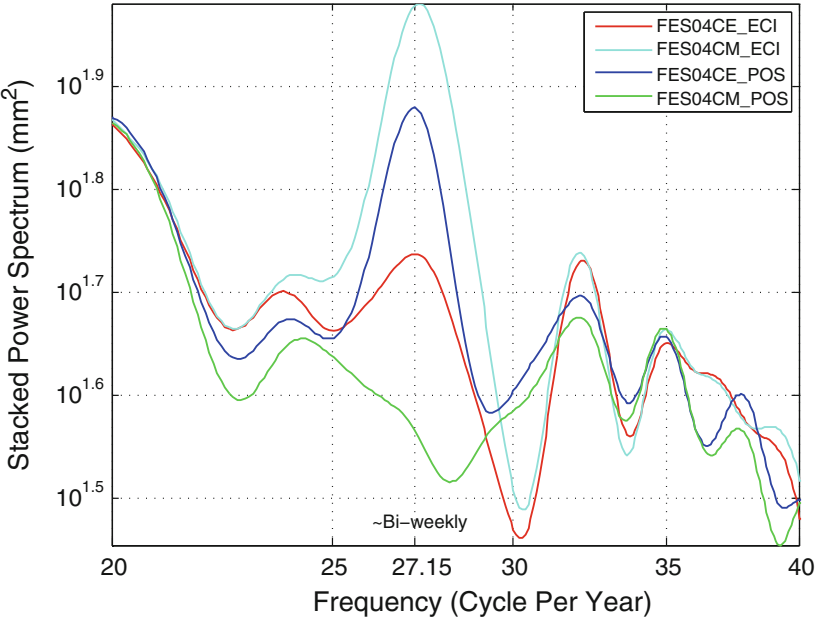

Fig. 6 Stacked power spectrum showing the $\sim 14$-day period component of 1-year detrended vertical coordinate timeseries for GPS solutions derived using two different orbit products: JPL's legacy products (eci), derived using OTL-CE coefficients, and JPL's reanalysis products (pos), derived using OTL-CM coefficients. For each set of orbits, we compare the noise spectra for solutions using OTL-CM and OTL-CE coefficients, giving four sets of solutions. The two solution sets with inconsistency in the OTL coefficients show significant noise peaks at $\sim 14$-day period, while the solutions using consistent OTL coefficients do not

2002 using two different orbit/clock products: JPL's legacy products (eci orbit) determined using OTL-CE, and JPL's reanalysis products (pos orbit) determined using OTL-CM. For each set of orbit and clock products, we compared solutions using OTL coefficients modeled in the CE and CM frames, giving us four different combinations of OTL coefficients used for the user and product solutions (Fig. 6). The ocean tide model FES2004 is adopted here. We label the solution sets based on the tide model, frame for OTL computations, and the orbit set used, for example FES04CM_POS means the FES2004 tide model, OTL computed in CM, and the reprocessed orbits (pos format).

Figure 6 displays the stacked power spectrum of the 14-day period component for the four different combinations. It is clear that the amplitude of the position variations at this period is smaller for the solutions in which the same OTL coefficients were used in the product solution and the user solution, FES04CM_POS and FES04CE_ECI. This confirms that significant spurious periodic signal will be introduced into the GPS position solutions if the user solution employs OTL coefficients that differ from those used in the product solution. The solutions using the reanalysis products, FES04CM_POS, display markedly lower noise compared to the solutions using the legacy products, FES04CE_ECI. Indeed, there are many differences between the two sets of orbit products. Thus, from this comparison alone we cannot be sure how much of the observed scatter is due to the use of any particular frame for computing the OTL coefficients. However, it is clear that a user's solution in point positioning 
mode needs to use OTL coefficients that are consistent with those used in the solution that generated the orbit and clock products. This confirms the suggestion of Scherneck et al. (2000) that such consistency should be very important.

We generated a 1-year (2006) set of solutions with orbits, clocks and positions estimated to test whether using one frame for OTL computations gives an intrinsically better result, using ocean tide model TPXO7.0. In a single-step global solution, there is no possibility of having an inconsistency in OTL coefficients as all parameters are estimated simultaneously in the solutions. We integrated orbits for each day based on the IGS reprocessed orbits (ig1), and estimated global solutions including adjustment to the orbits, using the same global stations as before. All processing models were the same as in the point positioning case, except that the solution was done in network mode with satellite clocks, orbit initial conditions and solar radiation pressure models estimated, along with all station parameters. We did not estimate Earth orientation parameters, but instead used IERS final values. One set of solutions used OTL-CM coefficients and the other set used OTL-CE, with no other differences.

We compared solutions by aligning each daily OTL-CE solution to the OTL-CM solution with a 7-parameter transformation and examining the residual differences after alignment. This comparison elucidates the distortion of the two solutions relative to each other, removing any differences that are described purely by translation, rotation or scaling of the network. The coordinate differences after transformation are very small, mostly $0.1-0.4 \mathrm{~mm}$ with a mean WRMS difference of $\sim 0.25 \mathrm{~mm}$ (Fig. 7, green bars). These differences are much smaller than the differences between the point positioning solutions (Fig. 7, red bars). Analysis of the stacked power spectrum (Fig. 8, top) indicates that the biases at $\sim 14$-day period shown in Fig. 6 clearly disappear. The magnitudes of the power for the global solutions, TPXO_CE and TPXO_CM differ at the level of $0.01 \mathrm{~mm}^{2}$ or less, so they are indistinguishable, and both are, in fact, very close to the best result in point positioning mode, FES04CM_POS. All these results suggest that the use of consistent OTL coefficients is more important than using one particular frame or the other; that is, an inconsistency between the product solution and user solution introduces larger errors than does using the wrong theory consistently. Our time series of global solutions is too short to demonstrate that using the correct theory (OTL$\mathrm{CM}$ coefficients) produces a superior result than consistently using OTL-CE coefficients, but we assume this to be the case.

\subsection{Network solutions with clocks estimated}

Many users do not employ point positioning mode, but rather fix orbits based on external products, and either estimate satellite clock errors or remove them by double-differencing. If the choice of frame for the OTL model computations

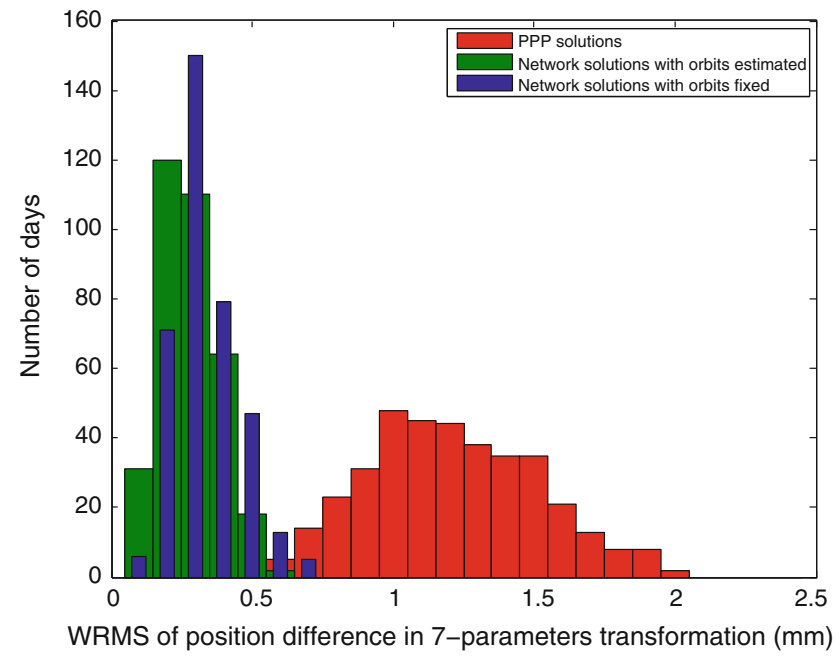

Fig. 7 Histograms of WRMS (mm) of vertical coordinate differences after application of a 7-parameter transformation between solutions with OTL modeled in two different frames. All the data are from the same year: 2006. Red bars represents the WRMS distortions for PPP solutions between FES04CE_POS and FES04CM_POS; green bars show WRMS distortions for network solutions with orbits, clocks and coordinates estimated, and blue bars for network solution with clocks and coordinates estimated

affects only the orbit product and not the satellite clocks, then this type of solution will exhibit the same biases as PPP solutions if the user solution uses OTL coefficients computed in a different frame. However, if the choice of OTL model coefficients mainly affects the clock parameters, the impact of inconsistency in this type of solution would be much smaller. To test the effects on this type of solution, we ran a 1-year (2006) series of network solutions with both clocks and positions estimated. JPL's reanalysis orbits were held fixed, but satellite clock parameters were estimated. We fixed a single reference clock, usually that at the site ALGO. The solutions were repeated using the each of the OTL-CM and OTL-CE model coefficients.

We used a 7-parameter transformation to compare the coordinate differences of the two different solution sets. The residual coordinate differences after transformation (Fig. 7, blue bars) have a mean WRMS of $\sim 0.3 \mathrm{~mm}$, very similar to what we observed for the global solutions with orbits estimated and much smaller than the differences we find for PPP solutions. The network solutions and PPP solutions differ only in that the satellite clocks are fixed to the values from the product solution in the PPP solution, but are estimated in the network solution. This result shows that the satellite clock estimates must be significantly different in solutions that use OTL-CM and OTL-CE, and that the difference is large enough to cause $\sim 1.3 \mathrm{~mm}$ distortions of the network when inconsistent OTL models are used in the user and product solutions. 
Fig. 8 Stacked power spectra showing the $\sim 14$-day period component from 1-year detrended vertical coordinate timeseries for global solutions with orbits, clocks and coordinates estimated (upper), and network solutions with clocks and coordinates estimated (bottom). FES04CM_POS (dashed line) is the same as that shown in Fig. 6 and is plotted here for comparison; it depicts the result in point positioning using JPL's reanalysis products (pos) and the OTL-CM model
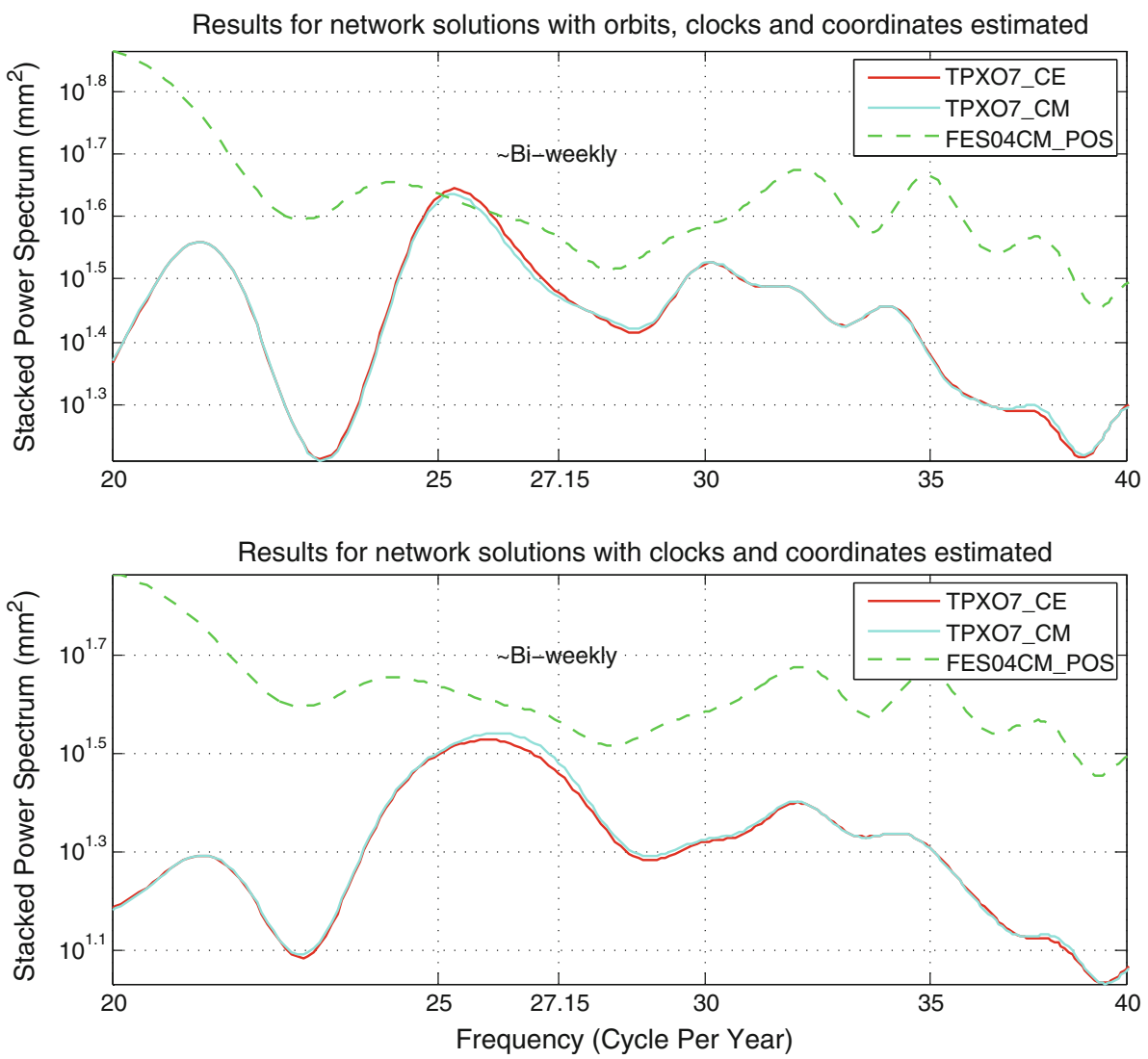

Figure 9 compares the frame parameters determined from transforming solutions using OTL-CE into alignment with solutions using OTL-CM (Fig. 7 compares the coordinate residuals after this transformation). The transformation parameters for network solutions with orbits, clocks and coordinates estimated (green line, Fig. 9) are smaller than other two solutions, being much less than $0.1 \mathrm{~mm}$. For the solutions that use fixed orbits (network solutions or PPP), the translation parameters are on the order of $0.3 \mathrm{~mm}$ or smaller, and the rotation parameters are equivalent to surface displacements of the same order. There is a small systematic bias of the $z$-axis rotation parameter for the PPP equivalent to a $\sim 0.3 \mathrm{~mm}$ displacement at the equator. The cause of this bias is unknown, but presumably it reflects a mean time bias that could impact time transfer. We do not observe any systematic geocenter offset between the two solution sets of a size equivalent to the magnitude of the center of mass correction (several $\mathrm{mm}$ ), because the geocenter translation of the daily frame is the geocenter offset averaged over the 24-h solution period, not the instantaneous offset at one epoch.

When all parameters are estimated simultaneously in a single solution, the differences between solutions using OTLCM and OTL-CE are of the order of $\sim 0.3 \mathrm{~mm}$, which are small, yet much larger than the GGOS goal, $0.1 \mathrm{~mm}$ accuracy at the global scale (Gross et al. 2009). The same level of network distortion is found for network solutions that used fixed orbits, although these also include frame biases equivalent to $\sim 0.3 \mathrm{~mm}$ displacements. PPP solutions have frame biases of the same order as the network solutions, but a much greater level of network distortion $(\sim 1.3 \mathrm{~mm})$. While the satellite orbit parameters must be different between solutions using OTL-CM and OTL-CE models, this difference is small enough that biases remain at the sub-millimeter level when inconsistent OTL models are used in the product and user solutions. The much larger degree of distortion in the PPP solutions means that the effect on the satellite clock parameters must be substantially larger than the effect on the orbits. This result seems counter-intuitive, but we suggest that it is because the bias introduced by inconsistent OTL models is similar for most stations that observe a given satellite at a given epoch. Each satellite is visible to stations only within a $\sim 66^{\circ}-76^{\circ}$ radius about the nadir point, depending on the elevation cutoff angle $\left(10^{\circ}-0^{\circ}\right)$. Stations on the opposite side of the Earth would have an opposite bias but do not observe the satellite. The common-mode part of the bias at each epoch (defined by the stations actually observing the satellite) will bias the satellite clock estimate for that epoch. In the limit of a sufficiently small regional network solution, the bias from inconsistent OTL models would be completely common mode, and would be removed by double-differencing or be indistinguishable from the satellite clock error. 
Fig. 9 Time series of frame parameters over a half-year period determined from a 7-parameter transformation between solutions using OTL-CM and OTL-CE models. [TX TY TZ] are translation parameters (in $\mathrm{mm}$ ); [RX RY RZ] represent rotation parameters (in $10^{-10} \mathrm{rad}$ ). The panel in (row 2, column 2) depicts the magnitude of the 3D translation vector (square root of $\left(\mathrm{TX}^{2}+\mathrm{TY}^{2}+\mathrm{TZ}^{2}\right)$ ). The red line is for transformation between FES04CE_POS and FES04CM_POS, the green line is for transformation of network solutions (orbits, clocks and coordinates estimated), and the blue line shows the results for network solutions (orbits fixed, satellite clocks and coordinates estimated)
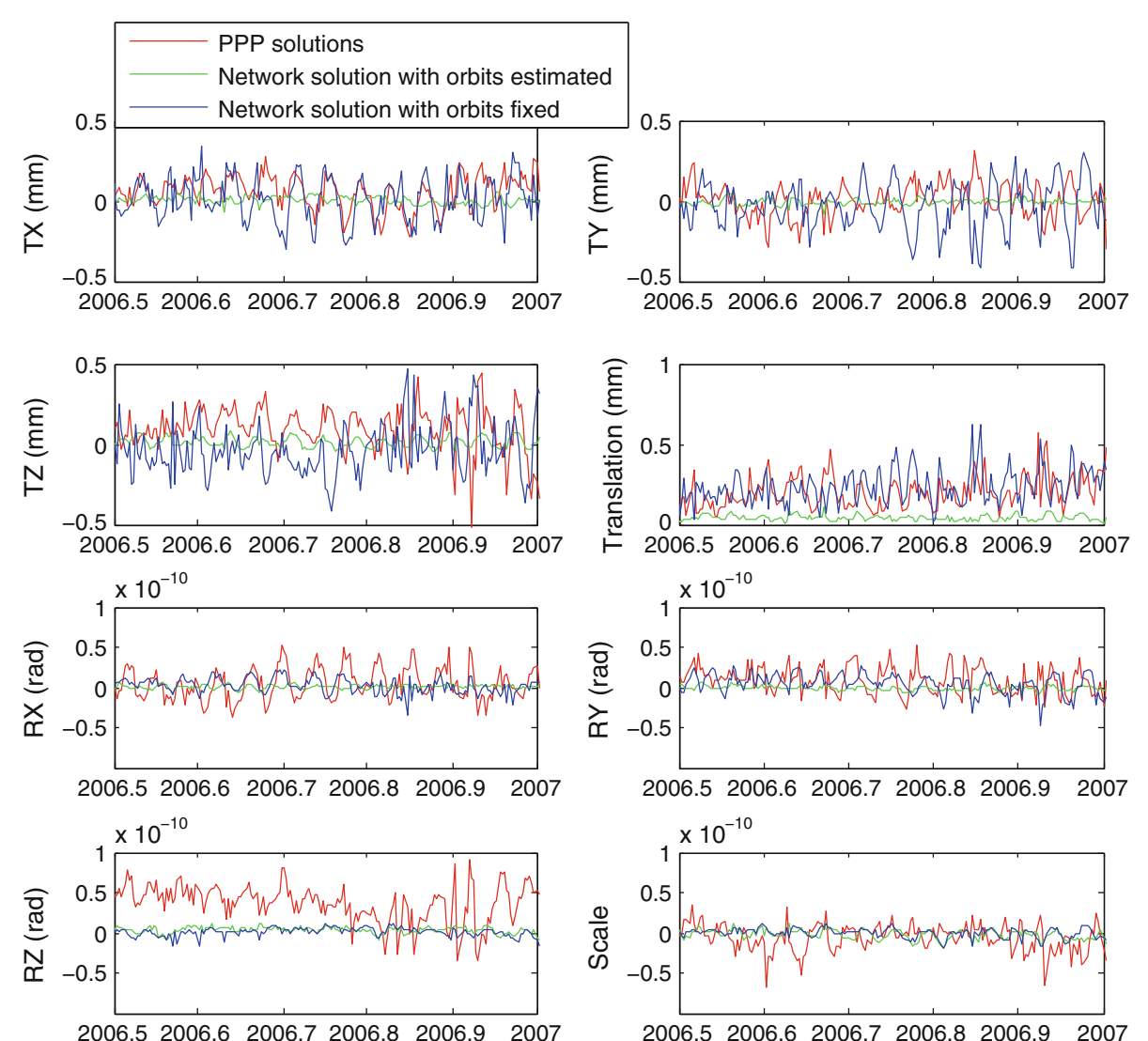

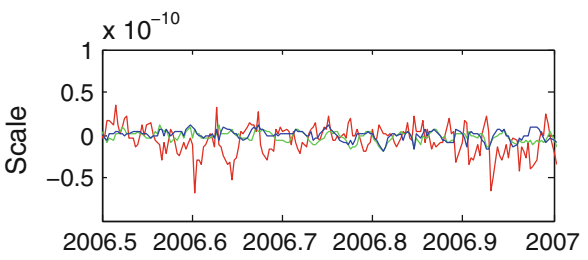

\subsection{Ambiguity resolution}

Tregoning and Watson $(2009,2011)$ found that ambiguity resolution affected the propagation of periodic modeling errors into station coordinates. Specifically, ambiguity-free solutions showed higher power than ambiguity-fixed solutions at the periods characteristic of tidal aliasing variations. To test the effect of ambiguity resolution on GPS coordinates with OTL corrections computed in inconsistent reference frames, we used the Ambizap algorithm to implement GPS carrier phase ambiguity resolution together with PPP (Blewitt 2008). Ambizap carries out ambiguity resolution on a baseline-by-baseline basis and applies the resulting coordinate changes to the combined PPP solution; the result is equivalent to a network-based ambiguity resolution. We ran a 1-yearlong solution series (calendar year 2006) using JPL's reanalysis orbit/clock products, but with OTL corrections computed in $\mathrm{CM}$ and $\mathrm{CE}$, respectively. We then compared solutions before and after ambiguity resolution with Ambizap.

We computed the vertical coordinate differences between OTL-CM and OTL-CE ambiguity-fixed solutions. A typical example (station TIDB) is given in Fig. 10 (top, blue line). Compared with the result of ambiguity-free solution (red line), the difference at 14-day period is still in the ambiguity-fixed result (blue line), although the amplitude is reduced slightly. However, for about $1 / 4$ of the stations the amplitude of the periodic difference was slightly increased. Overall, the ambiguity-fixed solutions show smaller differences at all frequencies, consistent with a lower overall noise level, but the reduction in power due to ambiguity resolution at the $\sim 14$-day period was smaller than at other periods (Fig. 10, bottom).

The stacked power spectra (Fig. 10, bottom) indicate that significant systematic errors are still introduced in ambiguity-fixed solutions if inconsistent OTL models are applied (red solid line). The smaller effect in ambiguity-fixed solutions is similar to that seen by Tregoning and Watson (2009, 2011) for atmospheric loading effects. However, the ambiguity-fixed solutions show the same essential characteristics as the ambiguity-free solutions, in that the use of inconsistent OTL models introduces significant systematic errors.

\section{Discussion}

\subsection{Theoretical interpretation}

GPS satellites are gravitationally attracted by the mass of the whole Earth system that includes the ocean, the atmosphere and other external and internal mass redistributions. The satellite trajectories are intrinsically determined with respect to the CM. Therefore, for consistency all correc- 

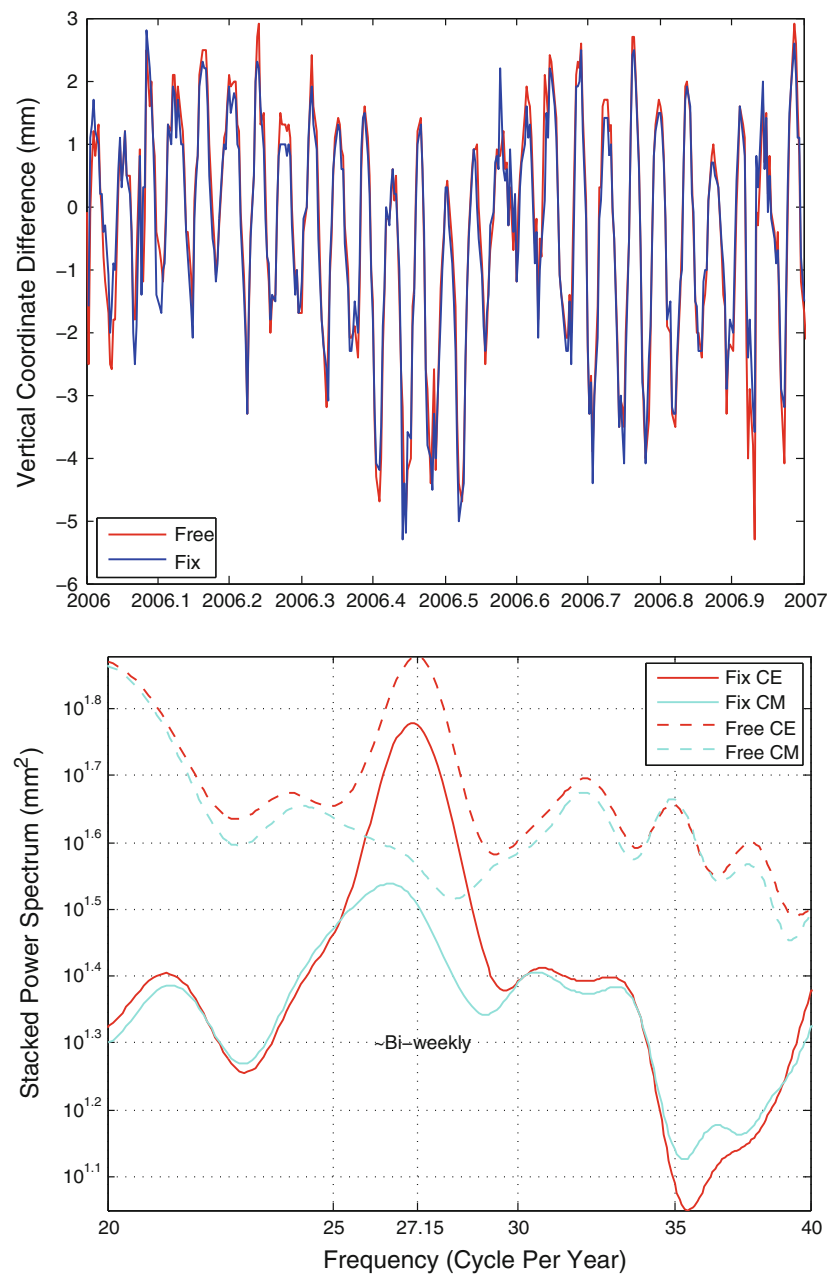

Fig. 10 Top Vertical coordinate difference between solutions using OTL-CM and OTL-CE, for ambiguity-fix solutions (blue), and ambiguity-free solutions (red). Bottom Stacked power spectra showing the $\sim 14$-day period component from 1-year detrended vertical coordinate timeseries; Solid lines are ambiguity-fixed result; dash lines are the same as FES04CE_POS and FES04CM_POS in Fig. 6, and for ambiguity-free solutions

tions applied in the coordinate model (especially loading corrections) ought to utilize the CM reference frame. Current IERS conventions require IGS Analysis Centers to use OTL-CM corrections when generating orbit/clock solutions (IERS Conventions 2010; Kouba 2009). Our study indicates that users need to use OTL coefficients that are consistent with those used by the analysis center that generated the orbit and clock products, especially when using point positioning. Using inconsistent OTL coefficients will introduce periodic systematic errors at the periods characteristic of aliased OTL, mainly $\sim 14$-days, $\sim$ semi-annual and $\sim$ annual periods. The GPS solutions themselves are biased at specific periods because, during the $24-\mathrm{h}$ positioning process, the frame bias does not exactly average to zero over the course of the time period of the GPS solution (Penna and Stewart 2003; Stewart et al. 2005; Penna et al. 2007). This bias is
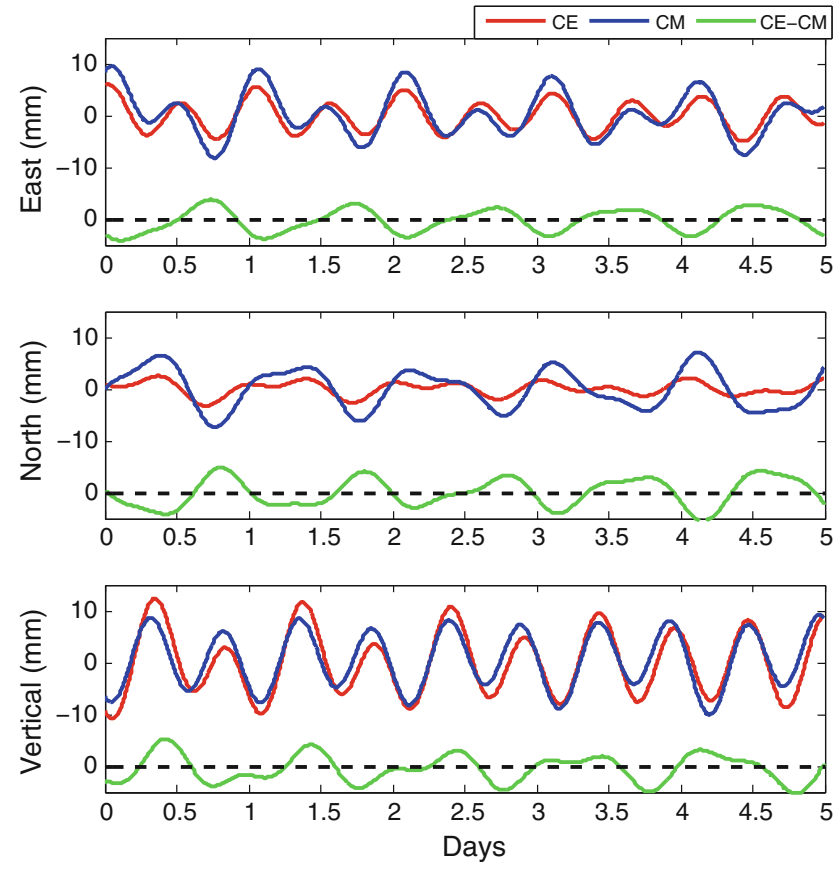

Fig. 11 Predicted OTL displacement of station TIDB for the first 5 days of 2005 (01/01/2005-01/05/2005). Displacements for OTLCE (red) and OTL-CM (blue) were calculated from ocean tide model TPXO7.0 using the SPOTL program, using the appropriate $\mathrm{CE}$ or $\mathrm{CM}$ Green's functions. The difference (CE-CM) is also plotted with a green line at the bottom of figures for each component, and illustrates the time-dependent bias introduced into the GPS observation model when inconsistent OTL coefficients are used in a processing strategy with orbits and clocks fixed to values estimated in a separate solution

over and above any errors that result from limitations of the tidal models themselves, which do not depend on the frame. Several recent studies have demonstrated that modern tidal models and OTL computation software produce very similar results (Thomas et al. 2007; Penna et al. 2008; Fok et al. 2010). The difference between solutions using OTL-CM and OTL-CE coefficients is much larger than the difference that results from using different tidal models.

Figure 11 shows predicted OTL displacements of station TIDB for the first five days of 2005. The displacements predicted by the OTL-CM and OTL-CE models are clearly different, and the differences are shown with a green curve. The difference curve (green) is the geocenter difference between the two frames, also termed the "center of mass correction" or "frame origin tides" (Scherneck et al. 2000), expressed in the local east-north-up coordinate system at this site. Because this difference results from a degree-1 deformation, OTL models for all sites around the world show a similar magnitude variation (in terms of the $3 \mathrm{D}$ vector). If one correction is applied in the solution that generated the orbit and clock products, and the other is applied in a user solution making use of those products, biases related to the green curve will be introduced into the GPS observation model, which will distort the estimated parameters in that solution. With a 24-h 
processing period, mismodeled tidal signals are propagated to longer-period noise in the GPS daily solutions (Penna and Stewart 2003; Penna et al. 2007), as revealed by the spectral analysis in Figs. 3 and 5. These biases distort the network (Fig. 7) and induce spurious variations in frame parameters (Fig. 9).

Scherneck et al. (2000) examined a set of point positioning solutions and compared observed tidal variations to models using OTL coefficients computed in both CM and CE. They expected to find the geocentric component in their solutions, but instead found that the observed variations agreed better with the OTL-CE model. They believed the reason for this to be that they used orbit and clock products generated from a solution generated using OTL-CE coefficients; thus the OTL-CM model was not consistent with the product solution. Our results show that their reasoning was correct. The orbit and clock products generated from a global solution essentially "lock in" the OTL model used in that product solution. Any user solution must use OTL coefficients consistent with the product solution. The choice of frame for the OTL model computations is much more important than the choice of tidal model. We find that the satellite clock parameters show larger differences than the orbit parameters, so the impact of inconsistencies between the product and user solutions is much larger for PPP solutions than for network solutions in which satellite clocks are estimated or removed by double-differencing.

\subsection{Effect of frame transformations}

The observed coordinate differences shown in Fig. 2 and analyzed in this paper demonstrate that introducing biases through inconsistency in OTL models causes distortions of the network that cannot be removed by a 7-parameter frame transformation (Fig. 7). Figure 12 shows vertical coordinate differences for TIDB from point positioning solutions, both before and after aligning the fiducial-free solution to ITRF2005. Before the frame alignment, each day's fiducialfree solution is in no particular frame, depending on the loose constraints applied in the solution that generated the orbit and clock products. Because we are comparing two solutions that are identical except for the OTL model applied, the two solutions being compared each day are nominally in the same frame. The patterns for the timeseries of the differences are very similar, and the amplitudes diminish slightly after the frame alignment. This confirms that most of the solution differences result from distortions in the network introduced by inconsistency in the OTL models. However, a portion of the bias from the OTL model frame error is absorbed into the frame transformation, and therefore into estimates of geocenter variation.

Tregoning and van Dam (2005) demonstrated that $80 \%$ of the geocenter motion can be recovered with a standard seven-

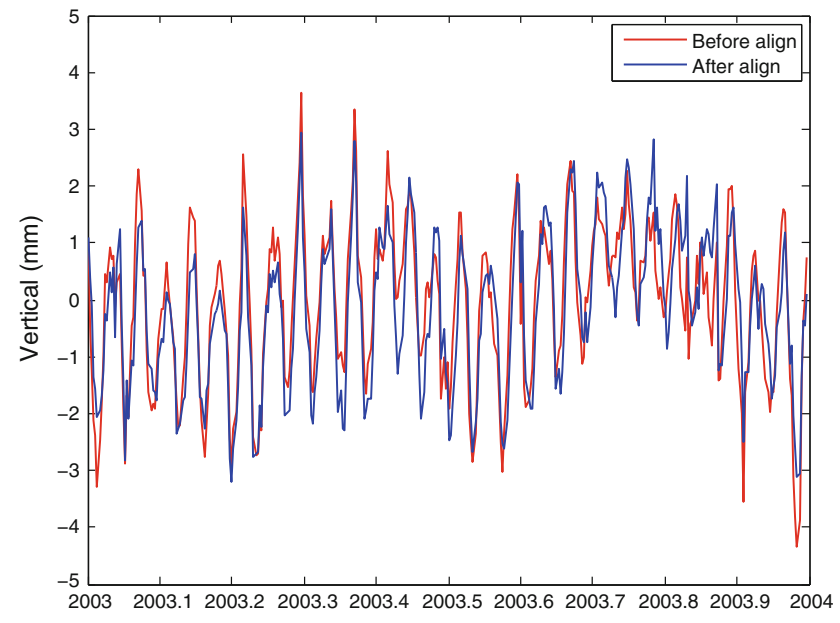

Fig. 12 Vertical coordinate differences between solutions using OTLCM and OTL-CE before aligning to ITRF2005 (red line), and after transforming to ITRF2005 (blue line), for station TIDB, for 2003. Most of the solution differences represent distortions of the network

parameter transformation for a global network, and the distribution of transformation sites can have a major impact on the transformation results. Thus, the globally distributed GPS sites used in this study improve our ability to distinguish surface displacements due to OTL from geocenter motion, during the process of transforming a non-fiducial solution into ITRF2005. If a small regional solution is used instead of a global solution, the coherence of the loading effect over long spatial wavelengths would not be preserved during the process of transformation. Subsequently, most of the coherent loading signals would be absorbed into the estimate of geocenter motion (Tregoning and van Dam 2005). This would impair the estimates for both the loading deformation and the geocenter.

\section{Conclusions}

Our study has shown that significant biases can be introduced into GPS solutions when a user solution uses OTL coefficients computed in a different reference frame to those used by the analysis center in their product generation solution. The choice of frame for the OTL model computations has a substantial effect on estimated satellite clock parameters, and a smaller effect on the estimated satellite orbits. For global solutions in which orbits, clocks and coordinates are estimated simultaneously, there are coordinate differences of $\sim 0.3 \mathrm{~mm}$ between solutions using OTL-CM and OTL-CE coefficients. When orbits and/or clocks determined from an external product solution are fixed, biases will be introduced into the user solutions if an OTL model is applied that is not consistent with the model used in the product solution. For network solutions the overall frame biases and distortion of 
the network remains at the sub-mm level, but for PPP solutions we find distortions of the network at the $\sim 1.3 \mathrm{~mm}$ level because the fixed satellite clocks are now inconsistent with the applied observation model.

Solutions in which inconsistent OTL coefficients are used display significant differences at several periodic components that are characteristic of aliasing of OTL errors. Power spectrum analysis for our 6-year detrended timeseries of continuous GPS positions shows that the most distinguishable difference occurs at a period of about $\sim 14$ days. Using JPL's current and reanalysis orbits products, which use OTL coefficients computed in the CM frame in accordance with IERS Conventions, we find a significant spectral peak at $\sim 14$-day period in the solution with OTL coefficients computed in the CE frame, but no peak at this period in the solutions using OTL computed in the CM frame. This result stands true for both ambiguity-free and ambiguity-fixed solutions. In addition, there are small differences at $\sim$ annual and $\sim$ semiannual periods. This phenomenon has been confirmed using both ocean tide models FES2004 and TPXO7.0, calculated using independent software. All of these improvements coincide well with the predicted periods of aliasing signals from OTL errors.

In any solution that uses fixed orbits or fixed orbits and satellite clocks, it is the analyst's responsibility to maintain consistency with the analysis center that generated the products. The need for consistency makes it critical both for analysis centers that generate orbit and clock products and researchers using these products to report which frame was used to compute the OTL coefficients in their analysis. Authors should also make clear which frame they used. The same is true for any other loading model applied at the observation level, such as the tidal component of atmospheric loading (Tregoning and Watson 2009, 2011). Both users and analysis centers that produce precise orbits and clocks should use OTL-CM coefficients for theoretical consistency and adherence to current IERS conventions. However, users of legacy products in which OTL-CE coefficients were used in the product generation will induce significant errors in their solutions if they do not maintain consistency and also use OTL-CE coefficients. This need for consistency may limit the degree to which PPP solutions can be used to assess other changes or improvements in observation models, such as the 2nd or higher order ionospheric effects (e.g., Kedar et al. 2003; Fritsche et al. 2005).

\footnotetext{
Acknowledgments We gratefully acknowledge Matt King and two anonymous reviewers for helpful suggestions that significantly improve this paper. We also thank Duncan Agnew for providing the SPOTL software, and Hans-Georg Scherneck for maintaining the web tool to compute OTL, both of which were critical for this study. Our CM Greens functions for SPOTL will be distributed with future SPOTL releases and are also available from the authors. This work was supported by NSF grants EAR-0911764 and EAR-0911677.
}

\section{References}

Agnew DC (1997) NLOADF: a program for computing ocean-tide loading. J Geophys Res 102:5109-5110

Amiri-Simkooei AR, Tiberius CCJM, Teunissen PJG (2007) Assessment of noise in GPS coordinate time series: Methodology and results. J Geophys Res 112:B07413. doi:10.1029/2006JB004913

Blewitt G (2003) Self-consistency in reference frames, geocenter definition, and surface loading of the solid Earth. J Geophys Res 108(B2). doi:10.1029/2002JB002082

Blewitt G (2008) Fixed point theorems of GPS carrier phase ambiguity resolution and their application to massive network processing: Ambizap. J Geophys Res 113:B12410. doi:10.1029/ 2008JB005736

Boehm J, Niell A, Tregoning P, Schuh H (2006) Global Mapping Function (GMF): A new empirical mapping function based on numerical weather model data. Geophys Res Lett 33:L07304. doi:10. 1029/2005GL025546

Boehm J, Heinkelmann R, Schuh H (2007) Short note: a global model of pressure and temperature for geodetic applications. J Geod. doi: 10 . 1007/s00190-007-0135-3

Dong D, Dickey JO, Chao Y, Cheng MK (1997) Geocenter variations caused by atmosphere, ocean and surface ground water. Geophys Res Lett 24:1867-1870

Dong D, Yunck T, Heflin M (2003) Origin of the international terrestrial reference frame. J Geophys Res 108(B4):2200. doi:10.1029/ 2002JB002035

Dragert H, James TS, Lambert A (2000) Ocean loading corrections for continuous GPS: a case study at the Canadian coastal site Holberg. Geophys Res Lett 27(14):2045-2048

Egbert GD, Erofeeva L (2002) Efficient inverse modeling of barotropic ocean tides. J Atmos Ocean Technol 19:183-204

Farrell WE (1972) Deformation of the Earth by surface loads. Rev Geophys 10:761-797

Fok HS, Iz HB, Shum CK, Yi Y, Andersen O, Braun A, Chao Y, Han G, Kuo CY, Matsumoto K, Song YT (2010) Evaluation of ocean tide models used for jason-2 altimetry corrections. Marine Geod 33(1 supp 1):285-303

Fritsche M, Dietrich R, Knöfel C, Rülke A, Vey S, Rothacher M, Steigenberger P (2005) Impact of higher-order ionospheric terms on GPS estimates. Geophys Res Lett 32:L23311. doi:10.1029/ 2005GL024342

Goad CC (1980) Gravimetric tidal loading computed from integrated Green's functions. J Geophys Res 85:2679-2683

Gross R, Beutler G, Plag HP (2009) Integrated scientific and societal user requirements and functional specifications for the GGOS. Global Geodetic Observing System 209-224

IERS Conventions (2010) Gérard Petit, Brian Luzum (eds) (IERS Technical Note; 36) Frankfurt am Main: Verlag des Bundesamts für Kartographie und Geodäsie, 2010. 179 pp., paperback, in press

Kedar S, Hajj GA, Wilson BD, Heflin MB (2003) The effect of the second order GPS ionospheric correction on receiver positions. Geophys Res Lett 30(16):1829. doi:10.1029/2003GL017639

King MA, Watson CS, Penna NT, Clarke PJ (2008) Subdaily signals in GPS observations and their effect at semiannual and annual periods. Geophys Res Lett 35:L03302. doi:10.1029/2007GL032252

Kouba J (2009) A Guide to Using International GNSS Service (IGS) Products. http://igscb.jpl.nasa.gov/components/usage.html

Penna NT, Stewart MP (2003) Aliased tidal signatures in continuous GPS height time series. Geophys Res Lett 30(23):2184. doi:10. 1029/2003GL018828

Penna NT, King MA, Stewart MP (2007) GPS height time series: shortperiod origins of spurious long-period signals. J Geophys Res 112:B02402. doi:10.1029/2005JB004047 
Penna NT, Bos MS, Baker TF, Scherneck HG (2008) Assessing the accuracy of predicted ocean tide loading displacement values. J Geod 82: 893-907. doi:10.1007/s00190-008-0220-2

Ray J, Altamimi Z, Collilieux X, van Dam T (2008) Anomalous harmonics in the spectra of GPS position estimates. GPS Solut 12: 55-64. doi:10.1007/s10291-007-0067-7

Scherneck HG, Johansson JM, Webb FH (2000) Ocean loading tides in GPS and rapid variations of the frame origin. Geodesy Beyond 2000-The Challenges in the First Decade vol. 121, Schwarz, Springer, Berlin 2000

Schmid R, Steigenberger P, Gendt G, Ge M, Rothacher M (2007) Generation of a consistent absolute phase center correction model for GPS receiver and satellite antennas. J Geod 81(12): 781-798. doi:10.1007/s00190-007-0418-y

Stewart MP, Penna NT, Lichti DD (2005) Investigating the propagation mechanism of unmodelled systematic errors on coordinate time series estimated using least squares. J Geod 79(8): 479-489. doi:10.1007/s00190-005-0478-6

Thomas ID, King MA, Clarke PJ (2007) A comparison of GPS, VLBI and model estimates of ocean tide loading displacements. J Geod 81:359-368. doi:10.1007/s00190-006-0118-9

Tregoning P, van Dam T (2005) Effects of atmospheric pressure loading and seven-parameter transformations on estimates of geocenter motion and station heights from space geodetic observations. J Geophys Res 110:B03408. doi:10.1029/2004JB003334
Tregoning P, Watson C (2009) Atmospheric effects and spurious signals in GPS analyses. J Geophys Res 114:B09403. doi:10.1029/ 2009JB006344

Tregoning P, Watson C (2011) Correction to "Atmospheric effects and spurious signals in GPS analyses". J Geophys Res 116:B02412. doi:10.1029/2010JB008157

Urschl C, Dach R, Hugentobler U, Schaer S, Beutler G (2005) Validating ocean tide loading models using GPS. J Geod 78: 616-625. doi:10.1007/s00190-004-0427-9

Vey S, Calais E, Llubes M, Florsch N, Woppelmann G, Hinderer J, Amalvict M, Lalancette MF, Simon B, Duquenne F, Haase JS (2002) GPS measurement of ocean loading and its impact on zenith tropospheric delay estimates: a case study in Brittany, France. J Geod 76(8): 419-427. doi:10.1007/s00190-002-0272-7

van Dam T, Wahr J, Chao Y, Leuliette E (1997) Predictions of crustal deformation and of geoid and sea-level variability caused by oceanic and atmospherc loading. Geophys J Int 129:507-517

Yuan LG, Ding XL, Zhong P, Chen W, Huang DF (2009) Estimates of ocean tide loading displacements and its impact on position time series in Hong Kong using a dense continuous GPS network. J Geod 83: 999-1015. doi:10.1007/s00190-009-0319-0

Zumberge JF, Heflin MB, Jefferson DC, Watkins MM, Webb FH (1997) Precise point positioning for the efficient and robust analysis of GPS data from large networks. J Geophys Res 102(B3):5005-5017 